\title{
Damage Detection in a Steel Beam Based on an Impact-Acoustics Approach
}

\author{
Eun-Taik Lee \\ Chung-Ang University, Seoul, South Korea. \\ Hee-Chang Eun \\ Kangwon National University, Chuncheon, South Korea.
}

\begin{abstract}
(Received 6 June 2015; accepted 28 June 2016)
Vibration and acoustics travel through a structure under the action of an impact force at a position on that structure. The acoustic detection system comprises either an accelerometer or a microphone to capture the acoustic signatures of the vibrations. Both signatures can be simultaneously collected by an impact hammer test and are analysed by this non-destructive test to obtain more reliable results than those of a single signature. This work investigates the damage identification of the beam structure based on the experimental data collected from the impact hammer test using an accelerometer and a microphone. The damage detection experiment on a steel beam illustrates the reliability of the defect detection using the simultaneous measurements by two sensors.
\end{abstract}

\section{INTRODUCTION}

The impact hammer test should be an effective nondestructive evaluation tool for investigating the structural state of health. Impact-acoustics are coupled with the structural vibration. The impact force causes the acoustic pressure as well as the structural motion to the structure.

A microphone should be a measurement sensor utilized for detecting damage from the acoustic pressure. Non-contact microphones measure acoustic pressure produced by the vibrating surface. Its disadvantage is a less accurate analysis of the velocity of the surface vibration. Due to a vertical point impact at the surface, the surface wave propagates horizontally near the free surface with dominant energy. Above the surface, the ground vibration due to the surface wave acts as an acoustic source to radiate an acoustic wave in the air. This acoustic radiation - a radiating surface wave - from the ground surface wave brings the same information about the dispersion properties with the frequency and wavelength.

The impact-acoustics method has many advantages in comparison with measurement with accelerometers. Many methods to provide non-contact damage detection methods have been developed. Lu et al. presented an integrated mobile acoustics sensing system, which was developed to estimate the thickness and the elastic modulus for pavement layers by collecting measurements continuously at a walking speed. ${ }^{1}$ Brigante and Sumbatyan reviewed acoustic non-destructive testing methods in the field of experimental studies of the physical properties of concrete. ${ }^{2}$ Kim et al. recognized that the impact force and the acoustic pressure data can be used to identify the presence of delamination. ${ }^{3}$ Luk et al. proposed a characteristic-extracting method using the wavelet packet decomposition for the impact acoustic non-detective evaluation. ${ }^{4}$ Tong et al. investigated the acoustics characteristics obtained from impact sounds excited by the impact on tilewalls and developed a non-destructive evaluation method for bonding integrity inspection. ${ }^{5}$ Klaerner et al. examined the elastic engineering constants and characterized the damping behaviour of the composites based on the acoustic analysis, tests of free oscillation of thin beams, and finite element models. ${ }^{6}$ Buck discussed the potential applications of the acous- tic non-destructive evaluation for characterizing and assessing structural inhomogeneities in varied materials. ${ }^{7}$ Ito and Uomoto experimentally observed the relation between the impact acoustics and the vibration at the same surface of the concrete. ${ }^{8}$ Kitagawa et al. provided a defect estimation formula for asphalt concrete, which was paved using the impact acoustics method. ${ }^{9}$ Zhu and Popovics proved the utility of the aircoupled impact-echo for the non-destructive evaluation of concrete under unwanted ambient acoustic noise. ${ }^{10}$ Hlavac verified the ability of the impact-echo method to detect a defect in a concrete structure. ${ }^{11}$ The impact-acoustics method could be applied when testing concrete and masonry structures. Zakiah et al. developed a non-invasive and non-destructive defect detection method for a seamless steel tube using the impact hammer method. ${ }^{12}$ Most methods using microphones to receive sounds and to analyse waveforms were developed to evaluate defects in concrete. The disadvantage of these methods is less accuracy in analyses of surface vibration velocity.

Due to their mass, accelerometers have a significant influence on the measured object. The mass of the accelerometer should be significantly smaller than the mass of the system to be monitored so that it does not change the characteristic of the object being tested.

Both the acoustic response (microphones) measured by the sound pressure and the structural response (acc) are collected. The Noise Transfer Function (NTF) measures the sound pressure with microphones via the mechanical excitation $(\mathrm{p} / \mathrm{F})$. The Vibration Transfer (VTF) measures the vibration in terms of acceleration over the excitation force $(\mathrm{a} / \mathrm{F})$. The experiment is performed with an impact hammer as a source, and the responses were also measured with microphones and accelerometers. Havranek dealt with a simply supported steel beam, producing a sound field and measured beam resonant frequencies with microphones and accelerometers and comparing their advantages. ${ }^{13} \mathrm{Wu}$ and Siegel investigated the fundamental principles of the accelerometer and the microphone measurement techniques. ${ }^{14}$ Silva et al. presented research results of the hammer test measured by an accelerometer and a microphone for a vibration analysis using non-invasive tests for fouling detection in pipelines. ${ }^{15,16}$ Donskoy et al. developed vibro-modulation 
and impact-modulation from the experimental observation of the modulation effect for various materials with various types of contact-type defects. ${ }^{17}$

Damage can be detected by evaluating the change in vibration signatures — such as radiated sound and vibration response - measured simultaneously at the same position. It can be expected that the signatures are changed in the neighbourhood of the damage region due to discontinuity of the traveling sound and motion, and it is utilized as an index to identify the damage. The existence of the damage leads to variations in the vibration amplitude and frequency using the output signals of the accelerometer and the microphone. The data measured by multiple sensors give more reliable results in detecting the damage than a single sensor.

This work investigates the reliability of damage detection by multiple sensors of a microphone and an accelerometer. It is shown that the measurement data from both sensors are sensitive to the external noise and the acoustic signals. Also, they are more sensitive to the noise than the acceleration signals. The following experiment illustrates that the measurement by multiple sensors provides more reasonable and explicit information on damage.

\section{FORMULATION}

A frequency response function (FRF) represents the relationship between the input and the output of a system. Considering the FRF between two points on a structure, it is measured by attaching an accelerometer or microphone at a particular point and exciting the structure at another point with a force gauge instrumented hammer.

\subsection{FRF of Structural Vibration $\left(F_{R F}{ }^{A}\right)$}

The dynamic behaviour of a structure, which is assumed to be linear and approximately discretized for $n$ degrees-offreedom (DOFs), can be described by the equations of motion:

$$
\mathbf{M} \ddot{\mathbf{u}}+\mathbf{C} \dot{\mathbf{u}}+\mathbf{K u}=\mathbf{f}(t)
$$

where $\mathbf{M}, \mathbf{K}$, and $\mathbf{C}$ are the $n \times n$ analytical mass, stiffness, and damping matrices, $\mathbf{u}=\left[u_{1} u_{2} \cdots u_{n}\right]^{T}$, and $\mathbf{f}(t)$ is the $n \times 1$ load excitation vector.

Substituting $\mathbf{u}(t)=\varphi q(t)$ into Eq. (1), pre-multiplying the result by $\varphi^{T}$, and normalizing the mode shapes to the unit modal mass $m_{i}(i=1,2, \cdots n)$ yields:

$$
\ddot{\mathbf{q}}+\Gamma \dot{\mathbf{q}}+\Lambda^{2} \mathbf{q}=\varphi^{T} \mathbf{f}
$$

where $\varphi_{i}^{T} \mathbf{M} \varphi_{\mathbf{i}}=1 ; \varphi_{\mathbf{i}}^{\mathbf{T}} \mathbf{M} \varphi_{\mathbf{k}}=0(i \neq k)$;

$$
\begin{gathered}
\boldsymbol{\Gamma}=\left[\begin{array}{cccc}
2 \omega_{1} \xi_{1} & 0 & \cdots & 0 \\
0 & 2 \omega_{2} \xi_{2} & \vdots & 0 \\
\vdots & \vdots & \ddots & \vdots \\
0 & 0 & \ldots & 2 \omega_{n} \xi_{n}
\end{array}\right] \\
\boldsymbol{\Lambda}^{2}=\left[\begin{array}{cccc}
\omega_{1}^{2} & 0 & \cdots & 0 \\
0 & \omega_{2}^{2} & \cdots & 0 \\
\vdots & \vdots & \ddots & \vdots \\
0 & 0 & \cdots & \omega_{n}^{2}
\end{array}\right]
\end{gathered}
$$

The next step is to establish the relationships between FRF and modal parameters for successful modal testing. Inserting
$\mathbf{q}=\mathbf{Q}(\Omega) e^{j \omega t}$ and $\mathbf{f}=\mathbf{F}(\Omega) e^{j \Omega t}$ into Eq. (2) and expressing it in the frequency domain yields:

$$
\left(-\Omega^{2} \mathbf{I}+j \Omega \boldsymbol{\Gamma}+\boldsymbol{\Lambda}^{2}\right) \mathbf{Q}(\Omega)=\varphi^{T} \mathbf{F}(\Omega) ;
$$

where $\Omega$ denotes the excitation frequency and $j=\sqrt{-1}$.

The $\mathrm{FRF}^{\mathrm{A}}$ matrix is obtained by inverting the left-hand side in Eq. (4) $\left(-\Omega^{2} \mathbf{I}+j \Omega \boldsymbol{\Gamma}+\boldsymbol{\Lambda}^{2}\right)$, pre-multiplying both sides of the result by $\varphi$, and using $\mathbf{U}(\Omega)=\varphi \mathbf{Q}(\Omega)$. Using the real eigenvalues and eigenvectors, the modal transformation leads to the representation of the FRF matrix for an excitation frequency $\Omega$ :

$$
\mathbf{H}(\Omega)=\sum_{i=1}^{n} \frac{\varphi_{i} \varphi_{i}^{T}}{\omega_{i}^{2}-\Omega^{2}+2 j \xi_{i} \omega_{i} \Omega} ;
$$

where $\mathbf{U}(\Omega)=\mathbf{H}(\Omega) \mathbf{F}(\Omega), \varphi$ is the transformation matrix, and $\mathbf{Q}(\Omega)$ is the modal displacement vector. An element $H_{k l}$ of the entire FRF $^{\mathrm{A}}$ matrix is:

$$
H_{k l}(\Omega)=\sum_{i=1}^{n} \frac{\phi_{k i} \phi_{l i}}{\omega_{i}^{2}-\Omega^{2}+2 j \xi_{i} \omega_{i} \Omega}
$$

describing the single output $Q_{k}$ due to a single dynamic input $F_{l}$.

\subsection{FRF $^{\mathrm{M}}$ of Sound Pressure}

The wave equation in an ideal fluid can be derived from hydrodynamics and the adiabatic relation between pressure and density. The acoustic wave propagation equation is:

$$
\Delta p=\frac{\partial^{2} p}{\partial x^{2}}+\frac{\partial^{2} p}{\partial y^{2}}+\frac{\partial^{2} p}{\partial z^{2}}=\frac{1}{c^{2}(x, y, z)} \frac{\partial^{2} p}{\partial t^{2}}
$$

where $p$ denotes the acoustic pressure of the wave propagation in the space $(x, y, z), c(x, y, z)$ is the local sound velocity, and $\Delta$ represents the Laplacian operator. The sound is produced by natural or artificial phenomena through a forced mass injection. It leads to the inhomogeneous wave equation:

$$
\Delta p-\frac{1}{c^{2}(x, y, z)} \frac{\partial^{2} p}{\partial t^{2}}=f(x, y, z, t) .
$$

Using the frequency-time transform of $p=P e^{i \omega t}$ and $f=$ $F e^{i \omega t}$, the wave equation leads to the frequency-domain wave equation:

$$
\Delta P+k^{2} P=F(x, y, z, \omega) ;
$$

where $k(x, y, z)=\frac{\omega}{c(x, y, z)}$ is the medium wavenumber at radial frequency $\omega$. Equation (9) can be expressed by a similar form as the dynamic equation of Eq. (4), and the $\mathrm{FRF}^{\mathrm{M}}$ matrix can be established by the measurement data collected from acoustic sensors.

\subsection{Experiment-Based FRF}

The measured data are collected as $\mathrm{FRF}^{\mathrm{A}}$ and $\mathrm{FRF}^{\mathrm{M}}$, which are the ratio of the response mode and the sound pressure of a system to its excitation force, respectively. The FRF response data can be experimentally obtained by roving the measurement sensors or the impact hammer. The FRF can be expressed as a function of the cross and auto spectra, which can readily be obtained from most multi-channel data acquisition systems. The cross spectrum is computed by multiplying the Fourier 
spectrum of a measured response by the complex conjugate of the Fourier spectrum of a known input:

$$
G_{x y}(\Omega)=F_{x}(\Omega) F_{y}^{*}(\Omega) ;
$$

where $G_{x y}(\Omega)$ is the cross spectrum, $\Omega$ is the excitation frequency, $F_{x}(\Omega)$ is the Fourier spectrum of a measured response, and ' $*$ ' is the complex conjugate. The auto spectrum is computed by multiplying the Fourier spectrum of the input by the complex conjugate of itself:

$$
G_{y y}(\Omega)=F_{y}(\Omega) F_{y}^{*}(\Omega)
$$

where $G_{y y}(\Omega)$ represents the auto spectrum. Based on structural motion and sound pressure, the FRF is then defined as the ratio of the cross and auto spectrum:

$$
H(\Omega)=\frac{G_{x y}(\Omega)}{G_{y y}(\Omega)}
$$

where $H(\Omega)$ represents the $\mathrm{FRF}^{\mathrm{A}}$ or $\mathrm{FRF}^{\mathrm{M}}$ to be collected experimentally.

FRF data provide more information than modal data, as the latter are extracted from a very limited frequency range related to resonance. The collection of the FRF data corresponding to a specific resonance frequency is complex; it can be overcome by taking the FRF data within a frequency range including the specific resonance frequency and importing the proper orthogonal decomposition (POD). The POD extracts the basis to decompose the data so that the projection of the data contains as much energy as possible. Proper orthogonal modes (POMs) constitute a set of optimal basis functions with respect to the energy content of the signal. The POM obtained from the PODs effectively extracts the principal component of a large DOF system or the complex physical phenomena. This work utilizes POM curves extracted from FRF data collected by the accelerometer and the microphone to detect and evaluate the location of damage. In the following section, the validity of the proposed impact-acoustics approach is illustrated using a beam test.

\section{BEAM TEST}

A steel beam in Fig. 1 is tested to detect the location of the damage by using the impact hammer, an accelerometer, and a microphone. The beam is $1200 \mathrm{~mm}$ in length and its gross cross-section is $50 \mathrm{~mm} \times 9 \mathrm{~mm}$. The damage is located at $930 \mathrm{~mm}$ between nodes 15 and 16 from the left end and its cross-section is $50 \mathrm{~mm} \times 6 \mathrm{~mm}$. The round bracket numbers in Fig. 1(a) indicate the node numbers (this work utilizes the node numbers without the round bracket). The experiment is carried out with the roving of an accelerometer and a microphone. The hammer is impacted at a single reference point to excite the beam, whereas the uniaxial accelerometer and the microphone roved around. The microphone and the accelerometer located under the hammer excitation at a fixed point simultaneously collect the measurement data of the acoustic and vibration signals. Nineteen measurement points are positioned at intervals of $60 \mathrm{~mm}$. Two different FRF data sets by the accelerometer and the microphone are collected at nineteen points $\left(H_{i, 11}, i=1,2, \cdots 19\right) . H_{i, 11}$ indicates the response at station $i$ due to a disturbing force at a stationary station 11 .

The measurement sensors and the impact hammer are shown in Fig. 2. The experiment is conducted using a DYTRAN model 3055B1 uniaxial accelerometer. A PCB model 426E01

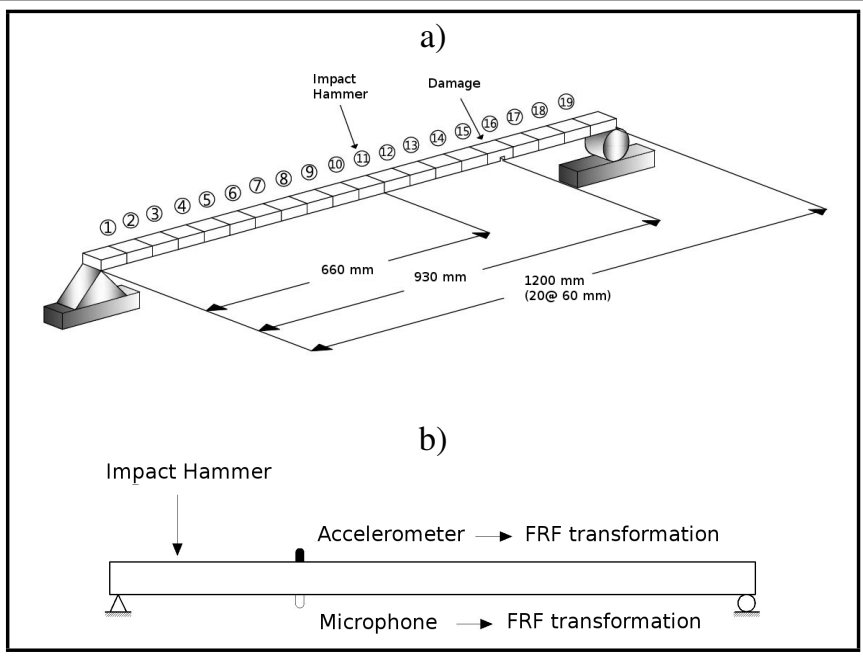

Figure 1. A beam structure: (a) A simply supported beam specimen and (b) installation of impact hammer and measurement sensors.

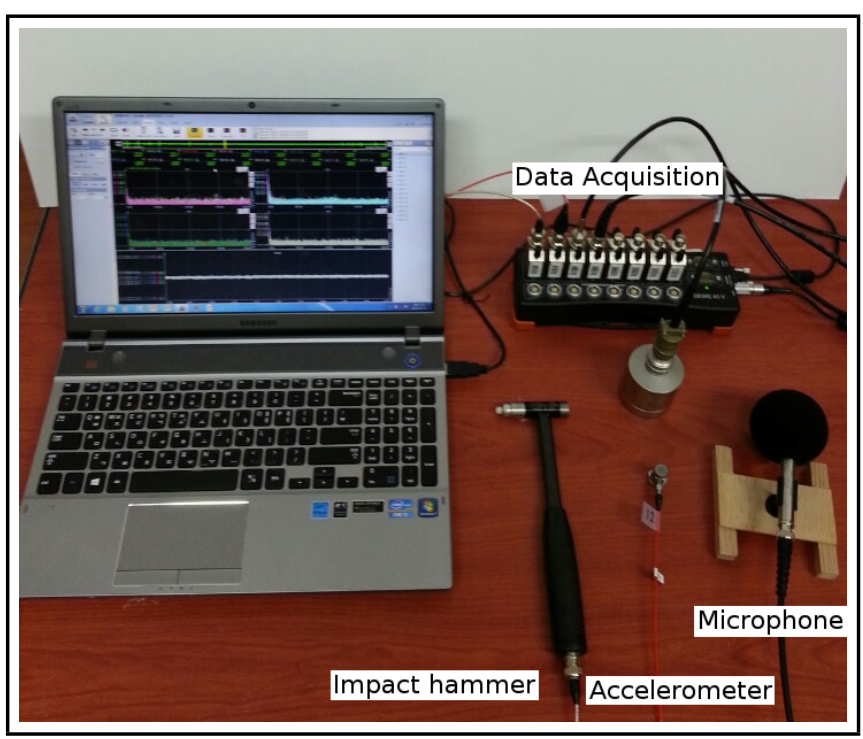

Figure 2. Equipment for the impact-acoustics test: the impact hammer, accelerometers, data acquisition, and microphone.

microphone preamplifier, and a miniature transducer hammer (Brüel \& Kjaer model 8204) to excite the system. The data acquisition system is a DEWETRON model DEWE-43.

The test is performed in a small room clad in acoustic insulation and noise insulation material to minimize external noise. Figure 3(a) and (b) represent the sound pressure in the test room and the acceleration responses measured on the beam caused by the external noises during a 10 minute span. The acoustic pressure represents more irregular responses to the external noise rather than the acceleration. This indicates that the microphone is more sensitive to the noise than the accelerometer.

Figure 4(a) and (b) exhibit the $\mathrm{FRF}^{\mathrm{A}}$ magnitude in $\mathrm{g} / \mathrm{N}$ and FRF $\mathrm{M}$ magnitude in $\mathrm{Pa} / \mathrm{N}$ measured by the accelerometer and the microphone, respectively, within the frequency range of $0-$ $20 \mathrm{~Hz}$. The plots represent the results measured at node 7 due to the impact at node 11 and exhibit that the first resonance frequency is located at $13.885 \mathrm{~Hz}$ on both sensors. The FRF ${ }^{\mathrm{A}}$ magnitude curve is more conservative than the FRF M curve because the acceleration-based experiment is less sensitive to the external noise than the microphone-based experiment as indicated in Fig. 3. The plots don't provide any information on 


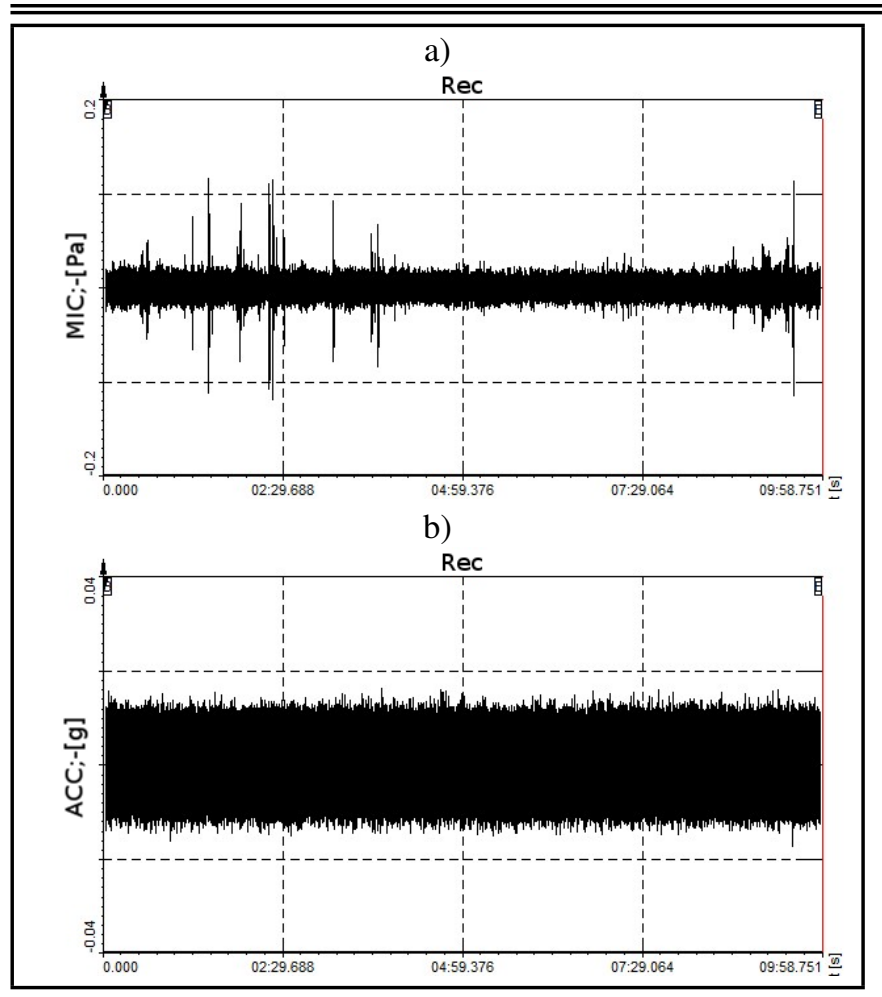

Figure 3. Noise test: a) External noise of sound pressure in a test room over 10 minutes; b) External noise of accelerations in a test room over 10 minutes.

the damage without the intact response data to compare with the measured data.

It is reported by Feeny and Kappagantu that the POMs represent the normal mode of vibration in undamped and lightly damped systems. ${ }^{18}$ The measured $\mathrm{FRF}^{\mathrm{A}}$ and $\mathrm{FRF}^{\mathrm{M}}$ data are transformed to the POM to obtain the principal component of optimal basis functions with respect to the energy content of the signal.

It's not easy to collect the FRF data at the moment of the first resonance frequency. Thus, FRF data sets in the neighbourhood of the first resonance frequency were selected to convert into POM. Three $\mathrm{FRF}^{\mathrm{A}}$ data sets and three $\mathrm{FRF}^{\mathrm{M}}$ data sets (corresponding to the frequency range less than the first resonance frequency) are extracted to estimate the POMs. Figure 5(a) displays the POM corresponding to the first POV estimated from the results utilizing the microphone. It is shown that the abrupt variation in the POM plot exists in the neighbourhood of node 16 where the damage is located. Figure 5(b) represents the POM extracted from the FRF ${ }^{\mathrm{A}}$ by the accelerometers; its abrupt variation is found in the neighbourhood of node 14 . The curvature of the POMs estimated by the $\mathrm{FRF}^{\mathrm{A}}$ at each location using a central difference approximation is given in Fig. 5(c). It is shown that its abrupt change is located in the neighbourhood of node 14. It can be concluded that the POM graphs taken by both sensors of the microphone and the accelerometer provide more reasonable and explicit information on the damage.

\section{CONCLUSIONS}

This study provides a method to detect damage in a steel beam using POMs that are extracted from the FRF data (measured simultaneously by a microphone as a non-contact sensor and by a accelerometer as a contact sensor). The measured FRFs in the neighbourhood of the first resonance frequency are converted into the POMs depending on measurement sen-

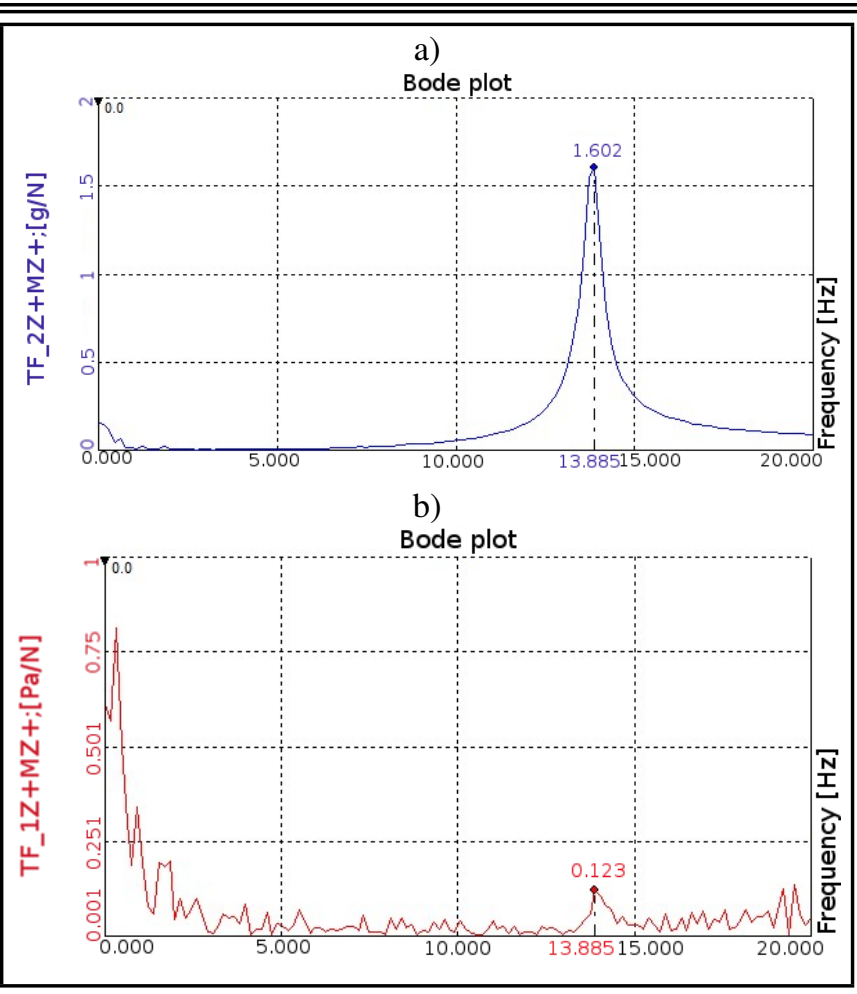

Figure 4. FRF curves: a) $\mathrm{FRF}^{\mathrm{A}}$ magnitude measured by a accelerometer and b) FRF $^{\mathrm{M}}$ magnitude measured by a microphone.

sors. It is observed that the damage is located at the region to represent the abrupt change in the POM curvature estimated by a central difference method. The acoustic signal data by the microphone is more sensitive to the external noise than the vibration signal by the accelerometer. It is recognized from the beam test that the measurement results utilizing multiple sensors provide more reliable and explicit damage information despite the external noise.

\section{ACKNOWLEDGEMENTS}

This research is supported by Basic Science Research Program through the National Research Foundation of Korea (NRF) funded by the Ministry of Education (2013R1A1A2057431).

\section{REFERENCES}

${ }^{1}$ Lu, Y., Zhang, Y., Cao, Y., McDaniel J. G., and Wang M. L. A Mobile Acoustic Subsurface Sensing System for Rapid Roadway Assessment, Sensors, 13, 5881-5896, (2013). https://dx.doi.org/10.3390/s130505881

2 Brigante, M. and Sumbatyan, M. A. Acoustic methods for the nondestructive testing of concrete: A review of foreign publications in the experimental field, Russian Journal of Nondestructructive Testing, 49, 100-111, (2013). https://dx.doi.org/10.1134/S1061830913020034

3 Kim, S. J., Ahn, S. M., Hwang, I. H., and Hong, C. H. Tapping test and analysis for damage detection, Proceedings of the 18 th International Conference on Composite Materials, (2011).

4 Luk, B. L., Jiang, Z. D., Liu, L. K. P., and Tong, F. Impact acoustic non-destructive evaluation in noisy environment based on wavelet packet decomposition, Proceedings of the 


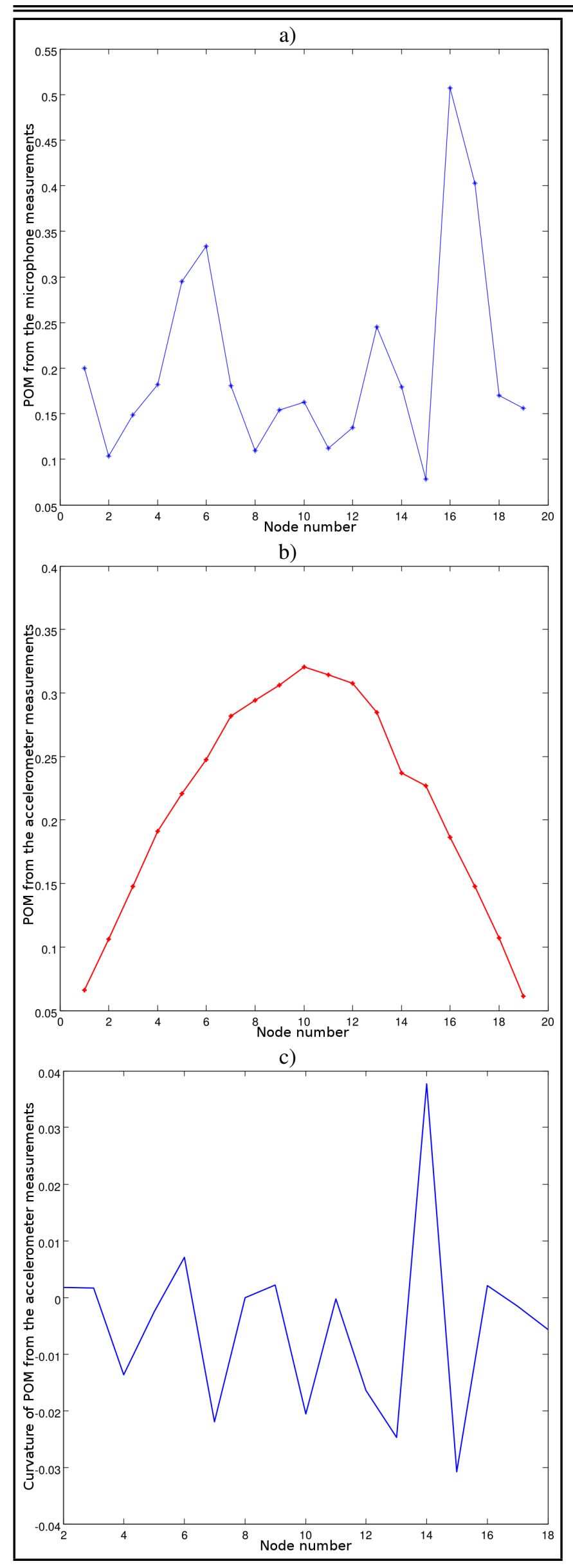

Figure 5. POM and its curvature curves: a) POM curve extracted from $\mathrm{FRF}^{\mathrm{M}}$, b) POM curve extracted from FRF $^{\mathrm{A}}$, and c) Curvature by POM curve extracted from $\mathrm{FRF}^{\mathrm{A}}$.
International MultiConference of Engineers and Computer Scientists, Hong Kong, (2008).

5 Tong, F., Tso, S. K., and Xu, X. M. Tile-wall bonding integrity inspection based on time-domain features of impact acoustics, Sensors and Actuators A:Physical, 132, 557566, (2006). https://dx.doi.org/10.1016/j.sna.2005.12.035

6 Klaerner, M., Marburg, S., and Kroll, L. Vibro-acoustic characterization of thermoplastic fibre reinforced composites, Proceedings of 20th International Congress on Acoustics, ICA, (2010).

7 Buck, O. Material characterization and flaw detection by acoustic NDE, JOM, 44, 17-23, (1992). https://doi.org/10.1007/BF03223166

8 Ito, Y. and Uomoto, T. Nondestructive testing method of concrete using impact acoustics, NDT \& E International, 30, 217-222, (1997). https://dx.doi.org/10.1016/S09638695(96)00059-X

9 Kitagawa, S., Kimura, S., and Moriyama, M. Deteriorated detection of the reinforced concrete slab of a highway bridge using impact acoustics method, 37th Conference on Our World in Concrete \& Structures, Singapore, (2012).

$10 \mathrm{Zhu}, \mathrm{J}$. and Popovics, J. S. Imaging concrete structures using air-coupled impact-echo, Journal of Engineering Mechanics-ASCE, 133, 628640, (2007). https://dx.doi.org/10.1061/(ASCE)07339399(2007)133:6(628)

11 Hlavac, Z. Detection of crack in a concrete element by impact-echo method, Ultragarsas, 64, 12-16, (2009).

12 Zakiah, A. H., Jamaludin, N., Syarif, J., and Yahya, S. Y. $\mathrm{S}$. Detection and analysis of defect in steel tube using vibration impact acoustic emission method, International Review of Mechanical Engineering, 8, 277-282, (2014).

13 Havranek, Z. Visualization of sound fields on vibrating steel beam using linear microphone array, Proceeding of the 6th WSEAS International Conference on Signal Processing, Computational Geometry \& Artificial Vision, Elounda, Greece, (2006).

$14 \mathrm{Wu}, \mathrm{H}$. and Siegel, M. Correlation of accelerometer and microphone data in the "Coin Tap Test." IEEE Transactions on Instrumentation and Measurement, 49, 493-497, (2000). https://dx.doi.org/10.1109/19.850382

15 Silva, J. J., Lima, A. M. N., Neff, F. H., and Neto, J. S. R. Vibration analysis based on hammer impact test for multilayer fouling detection, In XIX IMEKO World Congress Fundamental and Applied Metrology, Portugal, (2009).

16 Silva, J. J., Lima, A. M. N., Neff, F. H., and Neto, J. S. R. Vibration analysis based on hammer impact for fouling detection using microphone and accelerometer as sensors, Sensors and Transducers Journal, 112, 10-23, (2010).

17 Donskoy, D., Sutin, A., and Ekimov, A. Nonlinear acoustic interaction on contact interfaces and its use for nondestructive testing, NDT \& E International, 34, 231-238, (2001). https://dx.doi.org/10.1016/S0963-8695(00)00063-3

18 Feeny, B. F. and Kappagantu, R. On the physical interpretation of proper orthogonal modes in vibrations, Journal of Sound and Vibration, 211, 607-616, (1998). 